BULL. AUSTRAL. MATH. SOC.

VOL. 33 (1986), 389-395.

\title{
QUASI-CODIVISIBLE COVERS
}

\begin{abstract}
Paul E, BLAND
In this paper quasi-codivisible covers are defined and investigated relative to a torsion theory $(T, F)$ on $\operatorname{Mod} R$. It is shown that if $(T, F)$ is cohereditary, then a right $R$-module $M$ has a quasi-codivisible cover whenever it has a codivisible cover. Moreover, it is shown that if $(T, F)$ is cohereditary, then the universal existence of quasi-codivisible covers implies that the ring $R / T(R)$ must be right perfect. The converse holds when $(T, F)$ is pseudo-hereditary.
\end{abstract}

In [1], Bass has shown that a ring $R$ is right perfect if and only if every right $R$-module has a projective cover. Shortly thereafter, Wu and Jans [10] introduced the notion of a quasi-projective cover and showed that if a module has a projective cover, then it has a quasi-projective cover which is unique up to an isomorphism. The dual implication was investigated by Fuller and Hill [4]. They showed that the universal existence of quasi-projective covers implies that of projective covers, and hence that the ring must be right perfect.

In [3], the concept of a codivisible cover relative to a torsion theory $(T, F)$ on Mod $R$ was introduced and studied. Codivisible covers become projective covers when the torsion theory $(T, F)$ is selected to be the torsion theory in which every module is torsionfree. Rangaswamy [8] proved that if the torsion theory $(T, F)$ is pseudo-hereditary, then every right $R$-module has a codivisible cover if and only if $R / T(R)$ is a right perfect

Received 15 August 1985. This work was supported by Eastern Kentucky University's sabbatical leave program.

Copyright Clearance Centre, Inc. Serial-fee code: 0004-9727/86 $\$ A 2.00+0.00$. 
ring where $T(R)$ denotes the torsion ideal of $R$ with respect to $(T, F)$.

The purpose of this paper will be to define quasi-codivisible covers in such a fashion that when $(T, F)$ is the torsion theory in which every module is torsionfree, quasi-codivisible covers become the quasiprojective covers of $\mathrm{Wu}$ and Jans. It is then shown that if the torsion theory $(T, F)$ is cohereditary, that is, if $F$ is closed under taking factor modules, then a module with a codivisible cover has a quasicodivisible cover which is unique up to an isomorphism. It is also shown that the universal existence of quasi-codivisible covers implies that $R / T(R)$ is right perfect. Hence, the work of Wu and Jans, and Fuller and $\mathrm{Hi} 11$ can be obtained as a special case by selecting the torsion theory $(T, F)$ to be the torsion theory in which every module is torsionfree.

Throughout this paper, $R$ will denote an associative ring with identity and Mod $R$ will be the category of unitary right $R$-modules. The reader can consult [5], [6], or [9] for the terminology and standard results on torsion theories. $(T, F)$ will be a fixed torsion theory on Mod $R$ and $T(M)$ will denote the torsion submodule of $M$ with respect to $(T, F)$.

DEFINITION 1. A right $R$-module $M$ is quasi-codivisible if every row exact diagram of the form

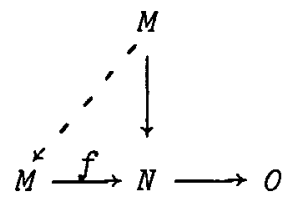

where $\operatorname{ker}(f) \in F$ can be completed commutatively.

DEFINITION 2. If $M$ is a right $R$-module, then a quasi-codivisible module $Q$ together with an $R$-linear epimorphism $\phi: Q \longrightarrow M$ is a quasicodivisible cover of $M$ if (i) $\operatorname{ker}(\phi)$ is small and torsionfree, and (ii) whenever $0 \neq T \subseteq \operatorname{ker}(\phi)$, then $Q / T$ is not quasi-codivisible. The following lemma will prove useful.

LEMMA 3. Let $\phi: Q \longrightarrow M$ be an epimorphism where $Q$ is quasicodivisible and $K=\operatorname{ker}(\phi) \in F$. If $K$ is stable under endomorphisms of $Q$, then $M$ is quasi-codivisible. 
Proof. Consider the row exact diagram

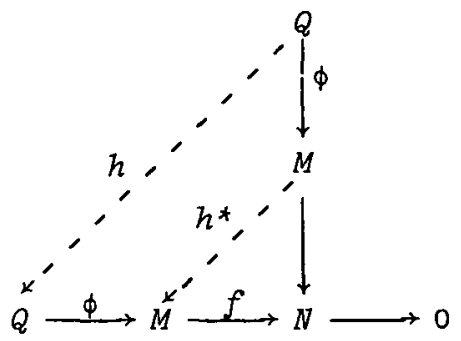

where $\operatorname{ker}(f) \in F$. If $H=\operatorname{ker}(f \circ \phi)$, then $f \circ \phi(T(H))=0$, and so $\phi(T(H)) \subseteq \operatorname{ker}(f)$. Hence, it follows that $\phi(T(H))=0$, and so $T(H) \subseteq \operatorname{ker}(\phi)$. Consequently, $T(H)=0$, and so $H$ is torsionfree. Thus, the outer diagram can be completed commutatively by an $R$-linear map $h$. But $h(K) \subseteq K$, and so we have an induced map $h^{*}$ which makes the inner diagram commutative.

LEMMA 4. If $(T, F)$ is cohereditary and $\phi: C \longrightarrow c / K: x \longrightarrow x+K$ is a codivisible cover of the quasi-codivisible module $C / K$, then $K$ is stable under endomorphisms of $C$.

Proof. Let $f$ be an endomorphism of $C$. Since the mapping $K \longrightarrow f(K): x \longrightarrow f(x)$ is an epimorphism and $K \in F$, it follows that $f(K) \in F$. Thus, since $K \oplus f(K) \longrightarrow K+f(K):(x, y) \longrightarrow x+y$ is an epimorphism, $K+f(K) \in F$. Now $f$ induces a map $f^{\star}: C / K \longrightarrow C /(K+f(K)): x+K \longrightarrow f(x)+K+f(K)$, and so consider the diagram

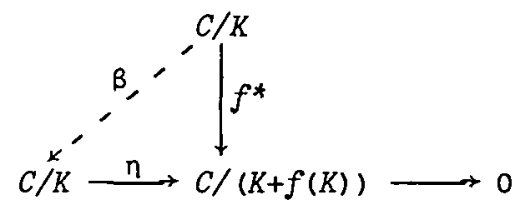

where $\eta(x+K)=x+K+f(K)$. The diagram can be completed commutatively by a map $\beta$, since $C / K$ is quasi-codivisible and $\operatorname{ker}(n) \in F$. Thus, using the codivisibility of $C$, we have a commutative diagram 


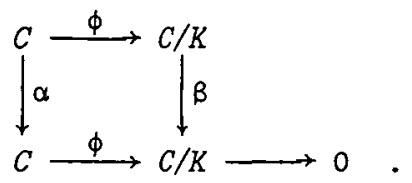

Now let $X=\{x \in C \mid f(x)-\alpha(x) \in K\}$. We claim that $X=C$. Since $\phi \circ \alpha(K)=\beta \circ \phi(K)=0, \alpha(K) \subseteq \operatorname{ker}(\phi)=K$, and so $\alpha$ induces a map $\alpha^{*}: C / K \longrightarrow C /(K+f(K)): x+K \longrightarrow \alpha(x)+K+f(K)$. Hence,

$$
\begin{aligned}
\left(f^{*}-\alpha^{*}\right)(x+K) & =f^{*}(x+K)-\alpha^{*}(x+K) \\
& =n \circ \beta(x+K)-(\alpha(x)+K+f(K) \\
& =n \circ \beta(x+K)-\eta \circ \phi \circ \alpha(x) \\
& =\eta \circ \beta(x+K)-n \circ \beta \circ \phi(x) \\
& =n \circ \beta(x+K)-n \circ \beta(x+K) \\
& =0 .
\end{aligned}
$$

Thus, $f^{\star}(x+K)-\alpha^{*}(x+K)=0$, and therefore $f(x)+K+f(K)-$ $(\alpha(x)+K+f(K))=0$. Consequently, $f(x)-\alpha(x) \epsilon K+f(K)$. Now let $f(x)-\alpha(x)=k_{1}+f\left(k_{2}\right), k_{1}, k_{2} \in K$. Then $f\left(x-k_{2}\right)-\alpha\left(x-k_{2}\right)=$ $k_{1}+\alpha\left(k_{2}\right) \in K$, since $\alpha\left(k_{2}\right) \in \alpha(K) \subseteq K$. Thus, $x-k_{2} \epsilon X$, and so $C=K+X$. But $K$ is small, and so $C=X$. Hence, if $x \in K$, then $x \in X$, and so $f(x)-\alpha(x) \in K$. But $\alpha(x) \in K$, and therefore $f(x) \in K$. This shows that $f(K) \subseteq K$.

PROPOSITION 5. If $(T, F)$ is cohereditary and if $M$ has a codivisible cover $\phi: C \longrightarrow M$, then $M$ has a quasi-codivisible cover $\phi^{*}: Q \longrightarrow M$ which is unique up to an isomorphism.

Proof. Use Zorn's Lemma and find the unique maximal submodule $X$ of $K=\operatorname{ker}(\phi) \quad$ which is stable under endormorphisms of $C$, and set $Q=C / X$. If $\phi^{*}: Q \longrightarrow M$ is the epimorphism induced by $\phi$, then $\operatorname{ker}\left(\phi^{*}\right)=K / X$. Now $K$ is small in $C$, and so $\operatorname{ker}\left(\phi^{*}\right)$ is small in $Q$ [7]. Note also that $\operatorname{ker}\left(\phi^{*}\right)$ is torsionfree, since $(T, F)$ is cohereditary. Hence, we have a map $C \longrightarrow C / X$ with $C$ codivisible and $X$ torsionfree and stable under endomorphisms of $C$. Hence, by Lemma $3, Q$ is quasicodivisible. Next, let $Y / X \subseteq \operatorname{ker}\left(\phi^{*}\right)$ be such that $(C / X)(Y / X) \cong C / Y$ is quasi-codivisible where $X \subseteq Y \subseteq K$. Then 
$0 \longrightarrow \mathrm{Y} \longrightarrow \mathrm{C} \longrightarrow \mathrm{C} / \mathrm{C} \longrightarrow \mathrm{O}$ is a codivisible cover of $C / Y$. Hence, it follows from Lemma 4 that $Y$ is stable under endomorphisms of $C$, and so it must be the case that $X=Y$. Thus, $\phi^{\star}: Q \longrightarrow M$ is a quasi-codivisible cover of $M$. Now let us show uniqueness. Suppose $\Phi: Q^{*} \longrightarrow M$ is a quasi-codivisible cover of $M$ and the consider the diagram

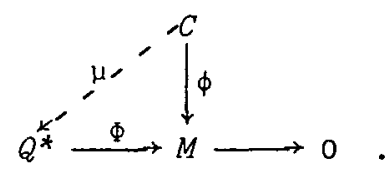

Since $\operatorname{ker}(\Phi)$ is torsionfree and $C$ is codivisible, the diagram can be completed commutatively, and so $\mu$ must be an epimorphism because ker $(\Phi)$ is small in $Q^{*}$. Now $\operatorname{ker}(\mu) \subseteq K$, and so it follows that $\mu: C \longrightarrow Q^{*}$ is a codivisible cover of $Q^{*}$. Hence, by Lemma $4, \operatorname{ker}(\mu)$ is stable under endomorphisms of $C$. Thus, if $X$ is as above, then $\operatorname{ker}(\mu) \subseteq X$. Suppose $\operatorname{ker}(\mu) \neq X$, then $\mu(X) \neq 0$. Now $\Phi \circ \mu(X)=\phi(X) \subseteq \phi(K)=0$, and so $0 \neq \mu(X) \approx \operatorname{ker}(\Phi)$. Now the map $\mu^{*}: C \longrightarrow Q^{\star} / \mu(X): y \longrightarrow \mu(y)+\mu(X)$ is clearly an epimorphism and we claim that $\operatorname{ker}\left(\mu^{*}\right)=X$. If $y \in X$, then $\mu(y)+\mu(X)=0$, and so $\mu^{*}(y)=0$. Hence, $X \subseteq \operatorname{ker}\left(\mu^{\star}\right)$. Now if $y \epsilon \operatorname{ker}(*)$, then $\mu(y)+\mu(X)=0$. Let $\mu(y)=\mu(x)$, so that $\mu(y-x)=0$. Then $y-x \in \operatorname{ker}(\mu) \subset X$, and so we see that $y \in X$, and therefore that $\operatorname{ker}\left(\mu^{\star}\right) \subseteq X$. Hence, $\operatorname{ker}\left(\mu^{*}\right)=X$. But this implies that $Q=C / X \cong Q^{\star} / \mu(X)$ which contradicts Definition 2 , since $\Phi: Q^{*} \longrightarrow M$ is a quasi-codivisible cover of $M$. Consequently, we must have $\operatorname{ker}(\mu)=X$, and so $\mu(X)=0$. But this yeidls $Q=C / X \cong Q^{*}$, and therefore $Q$ is unique up to an isomorphism.

PROPOSITION 6. If $(T, F)$ is cohereditary and every right R-module has a quasi-codivisible cover, then $R / T(R)$ is a right perfect ring. Moreover, if $(T, F)$ is pseudo-hereditary, then the converse holds.

Proof. Let $M$ be a right $R / T(R)$-module and suppose that $\theta: F \longrightarrow M$ is a free $R / T(R)$-module on $M$. Next, suppose that $\phi: Q \longrightarrow F \oplus M$ is a quasi-codivisible cover of $F \oplus M$. Since $(F \oplus M) T(R)=0, Q T(R) \subseteq \operatorname{ker}(\phi)$. Now Beachy [2] has shown that $Q T(R)=T(Q)$ when $(T, F)$ is cohereditary, and so $Q T(R)=0$, since $\operatorname{ker}(\phi)$ is torsionfree. Thus, $Q$ is an $R / T(R)$ module. Now consider the diagram 


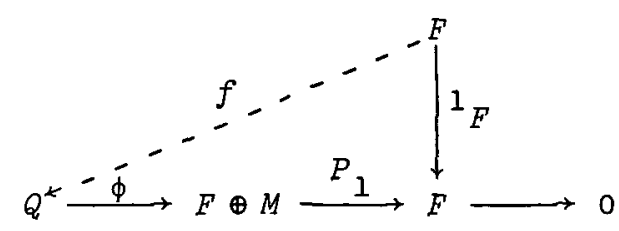

where $p_{1}$ is the first projection map and $f$ is the completing map given by the projectivity of $F$. If $M^{*}=\operatorname{ker}\left(p_{1}^{\circ} \phi\right)$, then we can assume that $Q=F \oplus M^{*}$. We claim that if $\phi^{*}=\left.\phi\right|_{M^{*}}$, then $\phi^{*}: M^{*} \longrightarrow M$ is an $R / T(R)$-projective cover of $M$. Clearly $\phi^{*}$ is an epimorphism with small kernel, and so we consider the diagram

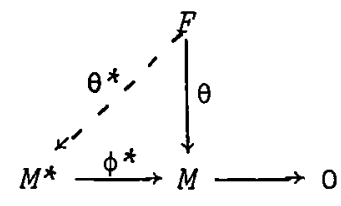

which completes commutativity by the projectivity of $F$. Note that $\theta$ * is an epimorphism, since $\operatorname{ker}\left(\phi^{*}\right)$ is small in $M^{*}$. Hence, we have a diagram

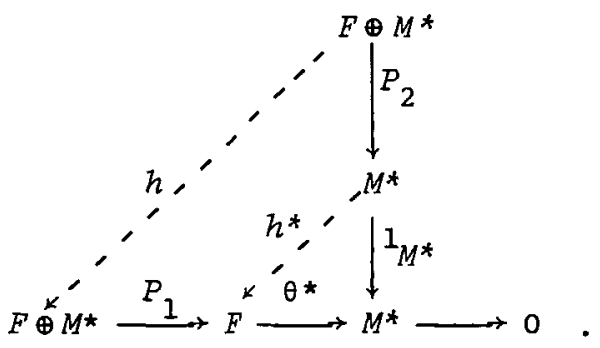

Since $0 \longrightarrow \operatorname{ker}\left(\phi^{*}\right) \longrightarrow M^{*} \stackrel{\phi^{*}}{\longrightarrow} M \longrightarrow 0$ is exact and $\operatorname{ker}\left(\phi^{*}\right)$ and $M$ are torsionfree $R$-modules, $i$ t. follows that $M^{*}$ is torsionfree because $F$ is closed under extensions. Consequently, $\operatorname{ker}\left(\theta * 0 p_{1}\right)$ is torsionfree, and so we have a completing map $h$. If $j_{2}: M^{*} \longrightarrow F \oplus M^{*}$ is the canonical injection and $h^{*}=p_{1} \circ h \circ j_{2}$, then the inner diagram is commutative, and so $M^{\star}$ is a projective $R / T(R)$-module. Thus, $\phi^{\star}: M^{\star} \longrightarrow M$ is an $R / T(R)$-projective cover of $M$, and thus $R / T(R)$ is right-perfect. 
For the converse, if $(T, F)$ is pseudo-hereditary, Rangaswamy [8] has shown that when $R / T(R)$ is right perfect, every right $R$-module has a codivisible cover. Hence, the result follows from proposition 5.

\section{References}

[1] H. Bass, "Finitistic dimension and a homological generalization of semi-primary rings", Trans. Amer. Math. Soc. 95 (1960), 466-488.

[2] J.A. Beachy, "Cotorsion radicals and projective modules", BulZ. Austral. Math. Soc. 5 (1971), 241-253.

[3] P.E. Bland, "Perfect torsion theories", Proc. Amer. Math. Soc. (1973), 349-353.

[4] K. Fuller and D. Hill, "On quasi-projective modules via relative projectivity", Arch. Math. (1970), 369-373.

[5] J. Golan, Localization of noncommutative rings (Marcel Dekker, New York, 1975).

[6] J. Lambek, Torsion theories, additive semantics, and rings of quotients (Lecture Notes in Mathematics, 177. Springer-Verlag, Berlin and New York, 1971).

[1] B. Pareigis, "Radikale und kleine moduln", Bayer. Akad. Wiss. Math. -Natur. Kl. Sitzungsber, 11 (1966), 185-199.

[8] K. Rangaswamy, "Codivisible modules", Comm. Algebra 2 (1974), 475-489.

[9] B. Stenstrom, Rings and modules of quotients (Lecture Notes in Mathematics, 237. Springer-Verlag, Berlin and New York, 1971).

[10] L. Wu and J. Jans, "On quasi-projectives", IZZinois J. Math. 11 (1967), 439-448.

Wallace 402 ,

Eastern Kentucky University,

Richmond, Kentucky 40475,

U.S.A. 